\title{
The Characteristics of Staphylococcus aureus Small Colony Variant Isolated from Chronic Mastitis at a Dairy Farm in Yunnan Province, China
}

\author{
Li-li Zhu, Feng-cai Zou, Yu-lin Yan, Qi-hui Wang, Yong-qiang Shi, and Wei-jie Qu \\ Institute of Animal Science and Technology, Yunnan Agricultural University, Kunming 650051, China \\ Correspondence should be addressed to Wei-jie Qu; 928701531@qq.com
}

Received 26 November 2015; Revised 20 January 2016; Accepted 20 January 2016

Academic Editor: Hassan Zaraket

Copyright @ 2016 Li-li Zhu et al. This is an open access article distributed under the Creative Commons Attribution License, which permits unrestricted use, distribution, and reproduction in any medium, provided the original work is properly cited.

Staphylococcus aureus is a major causative agent leading to bovine mastitis and has specific phonotypical characteristics including small colony, slow growth, and decreased hemolysis, therefore named as the small colony variants (SCVs). Out of 30 tested samples of the chronic S. aureus cases, one strain of SCVs (S. aureus SCV22) was isolated along with its parental strains (S. aureus11). S. aureus SCV22 showed a slow growth rate when it is compared with the parental strain. However, their resistant patterns were similar. Meanwhile, S. aureus SCV22 depicted the lower rate of apoptosis in bovine mammary epithelial cells. These findings of the present study presented the unique characteristics of S. aureus SCV22 for the first time in Yunnan province, which provided a prophase foundation for further study about the pathogenesis of S. aureus SCVs in chronic mastitis.

\section{Introduction}

Bovine mastitis is wildly prevalent among dairy cows mainly caused by the pathogenic Staphylococcus aureus, with a huge economic loss in dairy production. A hypothesis first proposed by Proctor et al. has showed that chronic or recurrent infection was associated with S. aureus SCVs [1]. Before the $S$. aureus SCVs were found, $S$. aureus was generally considered to be extracellular bacteria. However, many new reports showed that $S$. aureus SCVs owned the ability to persist intracellularly in nonprofessional phagocytes, such as epithelial cells, fibroblasts, osteoblasts, and endothelial cells $[2,3]$.

To the best of our knowledge, no reports have been documented for the isolation of S. aureus SCVs from dairy cows in Yunnan province, southwest China, as well as the related research. The aim of this study was to explore intracellular persistence, apoptosis of mammary epithelial cells, and establishment of mastitis model in S. aureus SCVs, and the results would provide prophase foundation to better know the infection mechanisms of $S$. aureus SCVs in chronic mastitis in further study.

\section{Materials and Methods}

2.1. Isolation and Identification of S. aureus and SCVs from Milk. Raw milk samples $(n=30)$ were aseptically gathered from scattered-feeding cows at a dairy farm in Kunming city of Yunnan province. Accordingly, all samples were cultured on Trypticase Soy Agar (TSA, HUANKAI, Guangzhou, China) complemented with 5\% sheep blood (Ruite, Guangzhou, China) and then cultivated at $37^{\circ} \mathrm{C}$ for $24 \mathrm{~h}$ and $48 \mathrm{~h}$, respectively. S. aureus was identified according to the phonotypical characteristics of being large, creamy, and forcefully hemolytic on TSA with $5 \%$ sheep blood. Potential SCVs colonies of tiny, nonpigmented, and nonhemolytic colonies on TSA with $5 \%$ sheep blood were also collected $[4,5]$. Isolates of potential SCVs were subcultured on TSA for ten generations to assess their stability. SCVs and their parental strains were primarily tested for routine biochemical properties; then the potential SCVs were identified as $S$. aureus SCVs with the gene (nuc, nucA, and 16srDNA) by multiple PCR amplification (Table 1). The identified S. aureus SCV and its parental strains were termed as S. aureus SCV22 
TABle 1: Primer information.

\begin{tabular}{|c|c|c|c|}
\hline Purpose gene & Sequence & Fragment length (bp) & $\overline{T_{m}}$ \\
\hline \multirow{2}{*}{$16 \mathrm{SrDNA}$} & Upstream primer: $5^{\prime}$-GGCGTTGCTCCGTCAGGCTT-3' & \multirow{2}{*}{375} & \multirow{6}{*}{$54^{\circ} \mathrm{C}$} \\
\hline & Downstream primers: $5^{\prime}$-CGCTGGCGGCGTGCCTAAT-3' & & \\
\hline \multirow{2}{*}{$n u c A$} & Upstream primer: $5^{\prime}$-CGCTTGCTATGATTGTGGTAGCC-3' & \multirow{2}{*}{239} & \\
\hline & Downstream primers: $5^{\prime}$-TTCGGTTTCACCGTTTCTGGCG-3' & & \\
\hline \multirow{2}{*}{ nuc } & Upstream primer: $5^{\prime}$-TCGTCAAGGCTTGGCTAAAGTTGC-3' & \multirow{2}{*}{126} & \\
\hline & Downstream primers: $5^{\prime}$-TCAGCGTTGTCTTCGCTCCAAA-3' & & \\
\hline
\end{tabular}

and S. aureus 11 , respectively. All isolates were stored at $-80^{\circ} \mathrm{C}$ in Trypticase Soy Broth (TSB, HUANKAI) complemented with $25 \%$ volumes of glycerin (HUANKAI) for further investigation.

2.2. Auxotrophism Assay. Auxotrophy test of the SCVs was performed on Mueller-Hinton agar (MHA, Difco BD, Beijing, China) with $1 \mu \mathrm{g} / \mathrm{mL}$ of hemin (Sigma, Shanghai, China), $1 \mu \mathrm{g} / \mathrm{mL}$ of thymidine (Sigma), $1 \mu \mathrm{g} / \mathrm{mL}$ of menadione sodium bisulfite (Sigma), and $100 \mu \mathrm{g} / \mathrm{mL}$ of thiamine as described previously $[6,7]$. Auxotrophism SCVs were identified, which showed the growth similar to their parental strains after incubating overnight at $37^{\circ} \mathrm{C}$.

2.3. Bacterial Growth Curves. SCVs and their parental strains were analyzed in triplicate for growth curve features $[1,8]$. The cryopreserved strains were inoculated into $10 \mathrm{~mL}$ TSB broth overnight at $37^{\circ} \mathrm{C} .1 \mathrm{~mL}$ of overnight culture was transferred to a flask containing $200 \mathrm{~mL}$ TSB and then incubated at $37^{\circ} \mathrm{C}$ on a rotary shaker $(150 \mathrm{rpm})$. Bacterial growth was determined by measuring the OD $600 \mathrm{~nm}$ per 4 hours.

2.4. Cell Culture. All reagents were purchased from Biological Industries (BI, Israel). The primary bovine mammary epithelial cells (Procell, Wuhan, China) were cultured in epidermal cell growth medium supplemented with $10 \%$ fetal calf serum (FCS, Sigma-Aldrich, Deisenhofen, Germany), epidermal growth factor $(0.1 \mathrm{ng} / \mathrm{mL})$, pig insulin, and hydrocortisone. The frozen cells were resuscitated in the cell culture plate with cell climbing piece in advance. The MECs were used after 3 passages for respective experiments.

2.5. Infection Experiments. For infection experiments [9], bacteria of $S$. aureus SCV22 and S. aureus11 were grown overnight at $37^{\circ} \mathrm{C}$ on TSA complemented with $5 \%$ sheep blood, picking a single colony to cultivate in liquid medium for $4 \mathrm{~h}$, then collecting bacteria, suspending them with DMEM/F12 (sigma), and then adjusting the thalli concentration to $10^{8} \mathrm{cfu} / \mathrm{mL}$. When the cells were grown to $80 \%$, one cell was infected using 100 bacteria. The infection test was terminated after $3 \mathrm{~h}$.

2.6. Apoptosis. The infected cells were dyed with Annexin VFITC/PI kit (Beyotime, Shanghai, China) [10]. The amount of apoptosis cells was measured by flow cytometry (BD FACSCalibur, USA) and analyzed with Cellquest Pro software.
2.7. Scanning Electron Microscopy. The infected cells were washed 5 times with PBS and the cells were fixed with glutaraldehyde phosphate buffer. Then cell dehydration was performed for $15 \mathrm{~min}$ in each solution using a graded ethanol from $30 \%$ to $100 \%$. The cell climbing was dried according to the critical point drying method before being sputter coated with gold in aE-1010 Ion Sputter Coater (Hitachi, Japan). Finally, the change of mammary epithelial cells was observed by A S-3000N scanning electron microscopy (Hitachi, Japan).

2.8. The Intracellular Persistence Assay. The infection experiment was conducted again and incubated for $3 \mathrm{~h}$ at $37^{\circ} \mathrm{C}$ with $5 \% \mathrm{CO}_{2}$ to allow for the phagocytosis and adhesion of $S$. aureus SCV22 and S. aureus 11 . The tested cell plate was washed with sterile PBS and $100 \mathrm{mg} / \mathrm{mL}$ of gentamicin and $50 \mathrm{mg} / \mathrm{mL}$ of penicillin were added, respectively. The cell plate was washed again and lysostaphin was added to eliminate extracellular bacteria and then incubated for $30 \mathrm{~min}, 3 \mathrm{~h}$, $9 \mathrm{~h}$, and $12 \mathrm{~h}$, respectively. At each time point, the cells were washed thrice with PBS to remove lysostaphin, which was followed by addition of $50 \mu \mathrm{L} 0.25 \%$ Triton X-100 to scatter epithelial cells and release intracellular bacteria. The cell lysing reagent was diluted with sterile water. $100 \mathrm{~mL}$ diluent was spread on the AGAR plate. The number of intracellular colony-forming units (CFU) was counted after $S$. aureus SCV22 and SASCV11 had grown overnight at $37^{\circ} \mathrm{C}$ at each time point.

2.9. Antimicrobial Susceptibility Testing. Antimicrobial susceptibility of $S$. aureus SCV22 was determined strictly with 10 antibiotics according to the drug susceptibility kit (Tianhe) instruction [11], while the standard S. aureus strain (ATCC29213, Tianhe, Hangzhou, China) was used as quality control (QC).

\section{Results}

3.1. Isolation and Identification of S. aureus11 and S. aureus SCV22 from Milk. Out of 30 samples collected from Holstein dairy cows, 8 isolates of suspected typical $S$. aureus11 were found. They were subcultured at $37^{\circ} \mathrm{C}$ for $16 \mathrm{~h}$ on TSA with $5 \%$ sheep blood. The result showed that all isolates have shown the typical morphology characteristics like $(4 \mathrm{~mm})$ $\beta$ hemolytic and creamy colonies (Figure 1(b)), and bacteria were Gram-positive coccus and arranged as grape-like clusters under light microscope. In addition, catalase test on pure TSA incubated at $37^{\circ} \mathrm{C}$ for $16 \mathrm{~h}$ was strong positive. Prevalence 


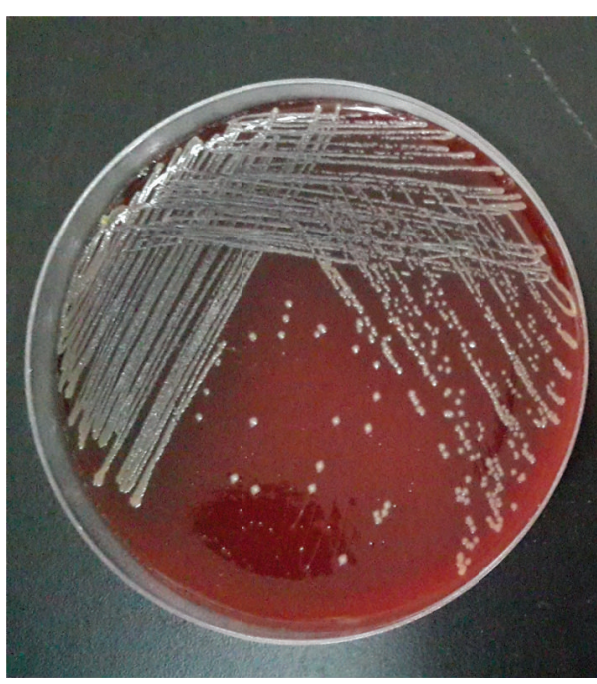

(a)

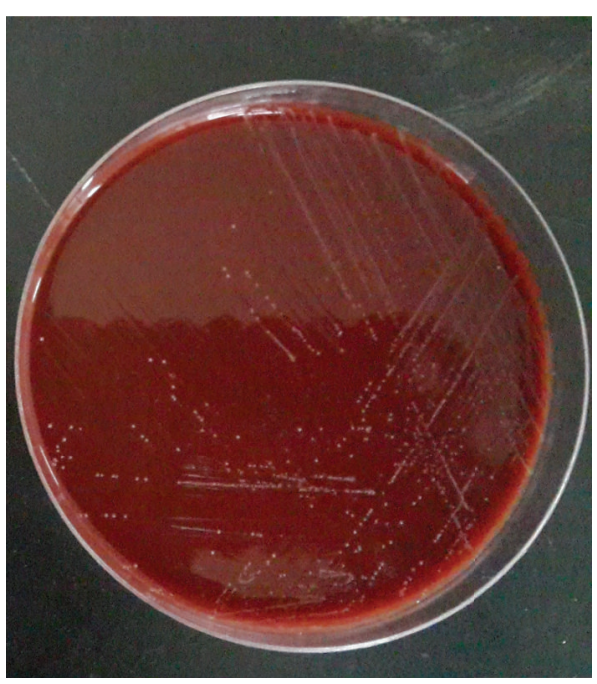

(b)

FIgURE 1: Colony morphological of S. aureus SCV22 with pinpoint colonies on Trypticase Soy Agar with 5\% sheep blood (a) compared to Staphylococcus aureus11 (b).

of coagulase was $100 \%$ by the commercial kit (Staphylase Test Kit, Oxoid, Beijing, China).

Out of eight $S$. aureus isolates, one was putative SCV22. Colonies of SCV11 with pinpoint $(0.3 \mathrm{~mm})$ were nonpigmented and failed to present an extremely slight hemolysis until incubated for $24 \mathrm{~h}$ at $37^{\circ} \mathrm{C}$ on TSA with $5 \%$ sheep blood (Figure 1(a)). Under the light microscope, these potential SCV11 appeared to be identical to typical $S$. aureus. For SCV11, catalase test was also positive, while coagulase was not detected. In the course of a continuous passage for ten generations these results were stable until the 10-generation passage.

All isolates were positive to multiple PCR test (Figure 2). The sequence analysis of $16 \mathrm{~S}$ rRNA showed that gene of $S$. aureus SCV22 and its parental strains are homologous with published S. aureus sequence.

3.2. Auxotrophism Assay. The auxotrophism analysis of $S$. aureus SCV22 revealed a thymidine dependence; the auxotrophism strains were close to their parental strains on MHA with $1 \mu \mathrm{g} / \mathrm{mL}$ of thymidine. However, the S. aureus SCV22 was negative for hemin, menadione, and thiamine (Figure 3).

3.3. Bacterial Growth Curves. According to the growth curves of $S$. aureus SCV22 and their parental strains (hereafter $S$. aureus11) cultured in pure TSB, $S$. aureusl1 showed a typical bacterial growth curve. In contrast, S. aureus SCV22 showed a linear growth curve, which had an extended lag phase, but the boundary between the lag phase and log phase could not be distinguished because of none breakpoint shown in Figure 4.

3.4. Intracellular Assay. Assays for S. aureus SCV22 and S. aureusll were done in triplicate to compare and quantify their ability to persist intracellularly within primary bovine mammary epithelial cells line. Measurement of CFU was

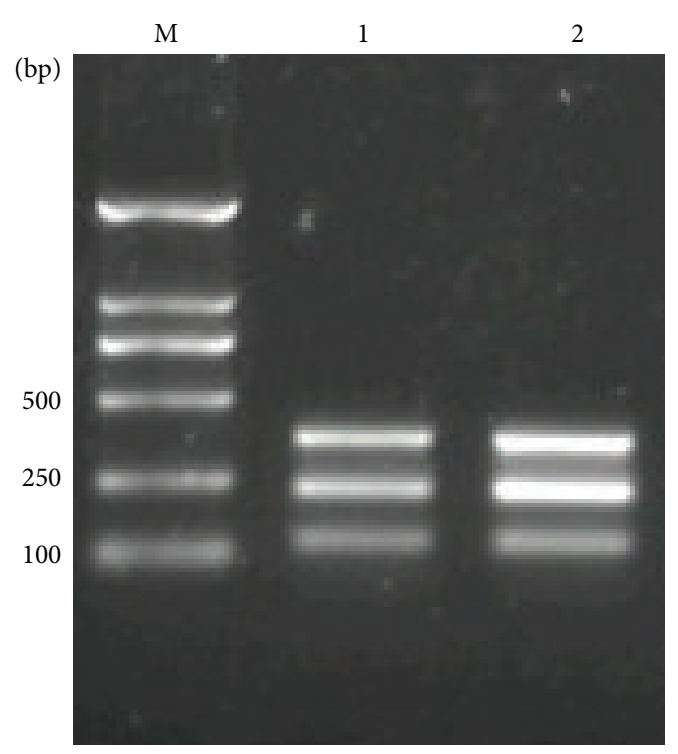

FIgURE 2: Molecular typing of S. aureus11 and S. aureus SCV22 isolated from cases of bovine mastitis by multiple Multiplex PCR. Molecular markers: 50 and 100 bp ladder (Takara, Japan).

conducted at the time points of $0.5 \mathrm{~h}, 3 \mathrm{~h}, 9 \mathrm{~h}$, and $12 \mathrm{~h}$, respectively (Figure 5). Hereby, prevalence of persistence from the time point of $3 \mathrm{~h}$ was becoming different between $S$. aureus SCV22 and $S$. aureus11, and the CFU number of $S$. aureus SCV22 was relatively stable (Figure 6).

3.5. Apoptosis. Compared to the control group, apoptosis rates of primary bovine mammary epithelial cells in infection groups of $S$. aureus SCV22 and S. aureus11 increased significantly $(P<0.05)$, and the difference between the $S$. aureus 


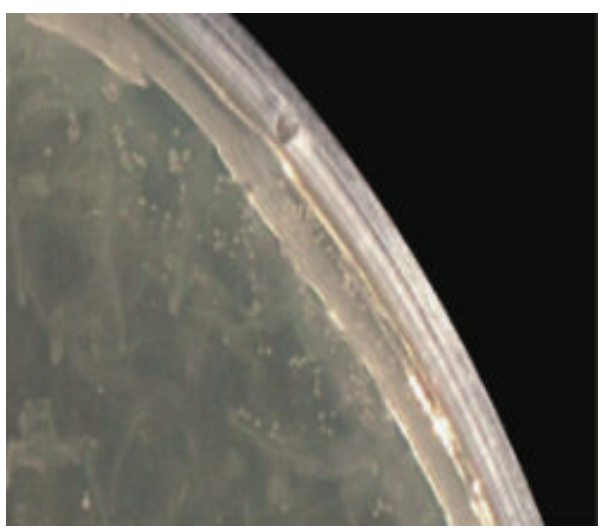

(a)

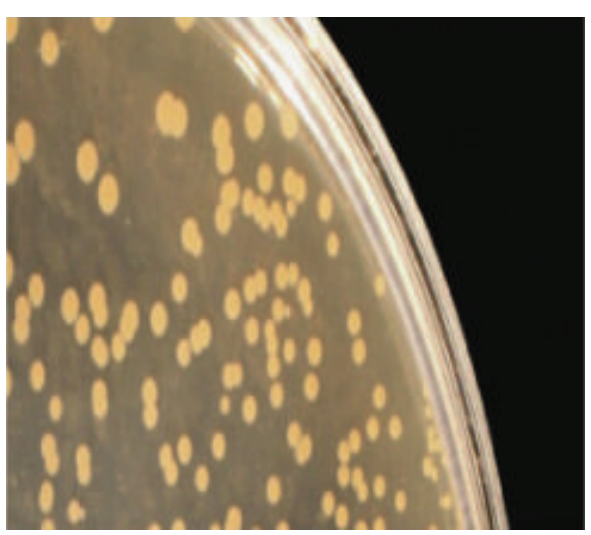

(b)

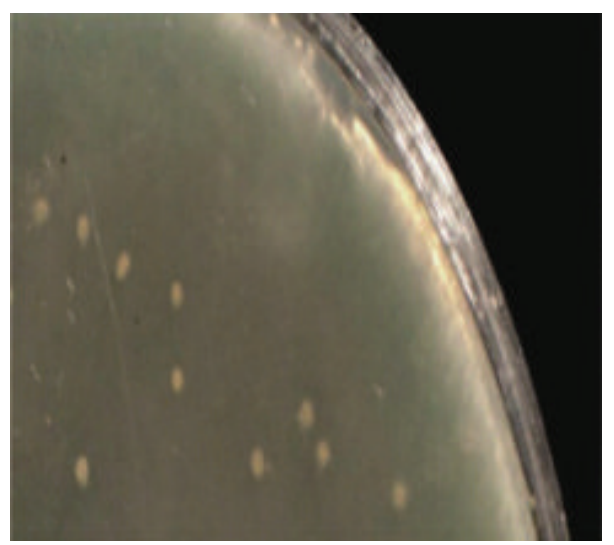

(c)

FIgURE 3: Auxotrophism assay: small colony variants S. aureus SCV22 (a), their parental strains (b), and auxotrophism strains (c).

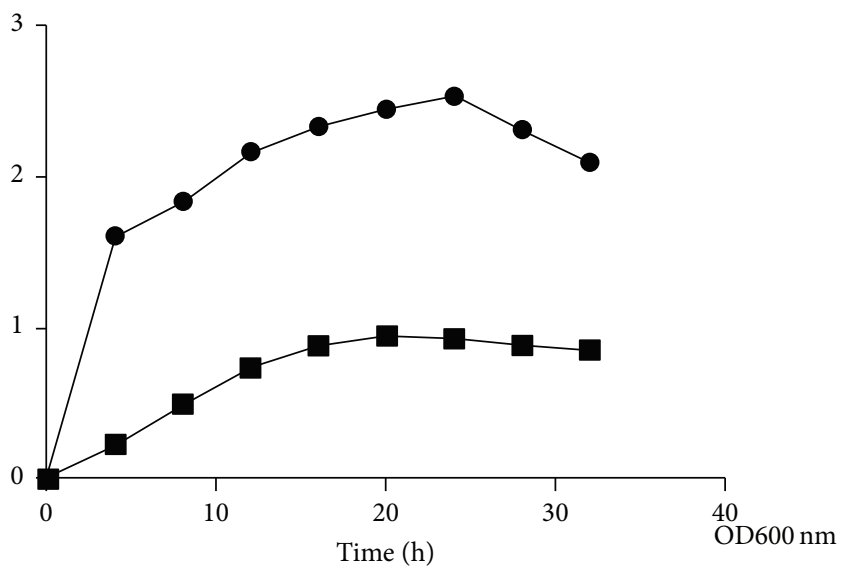

- S. aureus11

S. aureus SCV22

FIGURE 4: Growth curves of small colony variants S. aureus SCV22 in Trypticase Soy Broth compared to their parental strains.

SCV22 groups and the $S$. aureus1l groups was also statistically significant $(P<0.01)$ (Figure 7$)$.

3.6. Scanning Electron Microscopy. Under the electron microscopy, control cells were grown normally. In the
S. aureus11 infection groups, cells became round piece and flocked together after falling off, their cytomembrane ruptured, and the nucleus were unable to distinguish because of being shriveled. Cells of S. aureus SCV22 infection groups tended to flake, but cellular membrane was intact, and no extravasation of cytoplasm was found (Figure 8).

3.7. Antimicrobial Susceptibility Testing. The ATCC 25923 was sensitive to 10 antibiotics. On the contrary, the $S$. aureus SCV22 had a resistance to six antibiotics; the same results were given in repeated test (Table 2).

\section{Discussions}

Bovine mastitis is wildly common among most of the cow farms caused by $S$. aureus, especially the chronic mastitis. Many reports showed that a small colony variant from S. aureus with special phonotypical characteristics was the major pathogenic bacteria leading to chronic mastitis [12-14]. In the present study, although S. aureus SCV22 and S. aureus 11 had different phenotypic characteristics and growth curves, their targeted fragments were the same by multiple PCR amplification.

Since SCVs phenomenon was found from Eberthella typhosa strains by Jacobsen in 1910 [15], various SCVs from different bacteria have been reported [16-19]. Among them, 


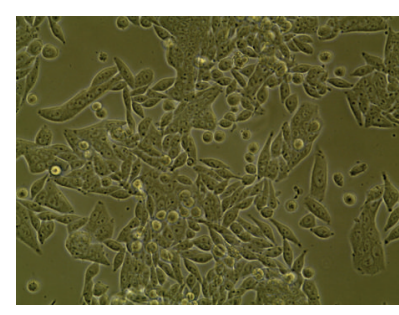

(a1)

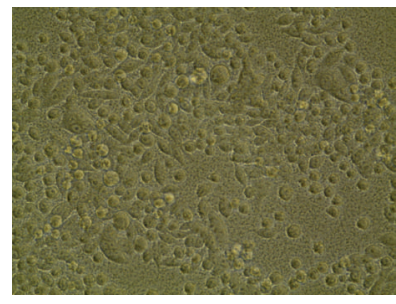

(a2)

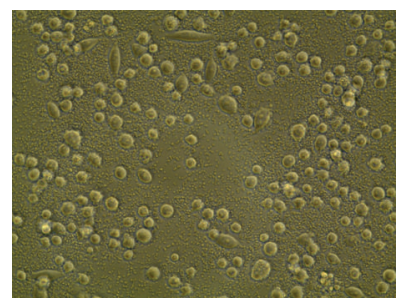

(a3)

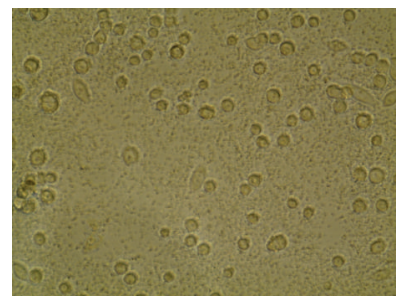

(a4)

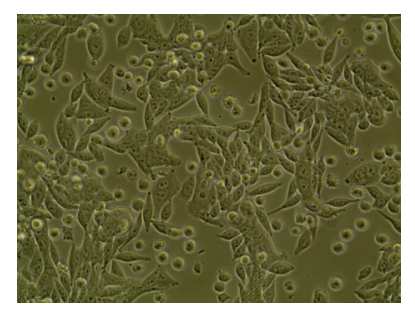

(b1)

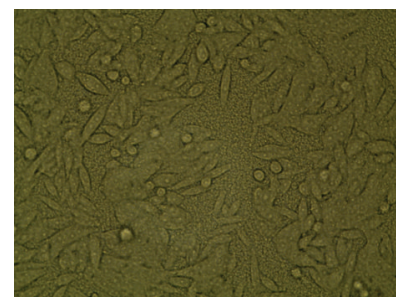

(b2)

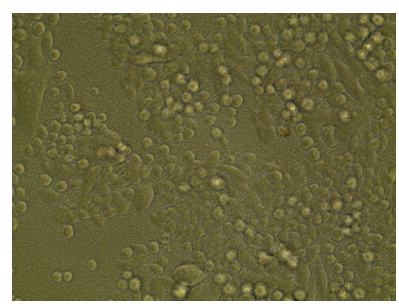

(b3)

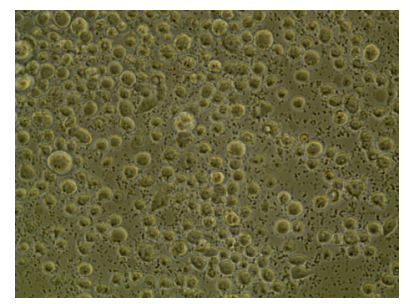

(b4)

Figure 5: (40x) Morphological changes indicating cell damage, detachment, and rounding of primary bovine mammary epithelial cells infected with small colony variants $S$. aureus SCV22 and their parental strains after 0.5 hours (a1, b1), 3 hours (a2, b2), 9 hours (a3, b3), and 12 hours (a4, b4). S. aureus11 was presented in (a1), (a2), (a3), and (a4); S. aureus SCV22 was presented in (b1), (b2), (b3), and (B4).

S. aureus SCVs were wildly studied; their main characteristics are small colony, slow growth, forming biofilms, surviving in host cells chronically, and even reducing the sensitivity to antibiotics, which are consistent to our results, of which small phenotype and slow growth of SCVs are mainly caused by metabolic defects [10], and two types can be divided, including electron transfer defect and thymine synthesis defect. The former is the typical auxotroph; the normal phenotype can be formed by supplying menadione or hemin. In this study, the result showed that $S$. aureus SCV22 was thymine-dependent $S$. aureus SCVs; this type had been reported from patients with cystic fibrosis (CF) previously.

In the present study, $S$. aureus SCV22 had a high resistance to SXT, while their parental strains were sensitive. SXT can interfere with the synthesis of tetrahydrofolic acid to inhibit the growth of S. aureus [4]. In order to resist SXT, thymine-dependent SCVs absorbed the thymine in extracellular and survived ultimately. This phenomenon of thyminedependent SCVs may be related to gene disruption mutation of thyA, because the thymidylic acid synthetase and tetrahydrofolic acid encoded by gene are important coenzyme for thymidylic acid synthetase.

In this study, the results of intracellular assay, apoptosis test, and scanning electron microscopy indicated that $S$. aureus SCVs persisted longer in nonprofessional phagocytes without profound damage, as previously reported [20-22]. This feature successfully avoided the host immunity and antibiotic therapy that partly explained its antibiotic resistance and the failure of antibody-mediated immune response (AMIR).

In conclusion, chronic mastitis is widespread in cow farms in Yunnan probability due to free-ranging, outdated 


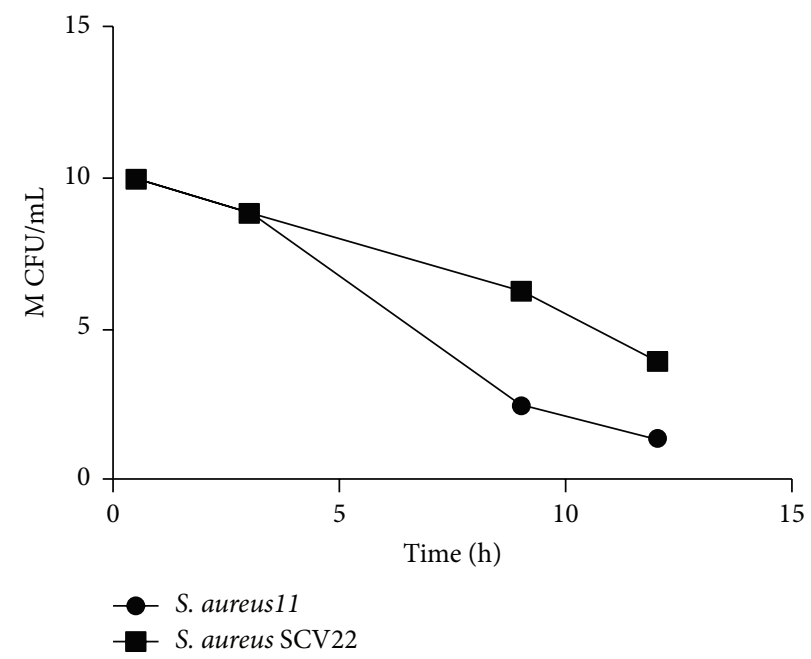

FIGURE 6: S. aureus SCV22 M CFU/mL (1 million colony-forming units/mL) recovered from primary bovine mammary epithelial cells at several time points compared to $S$. aureusll.
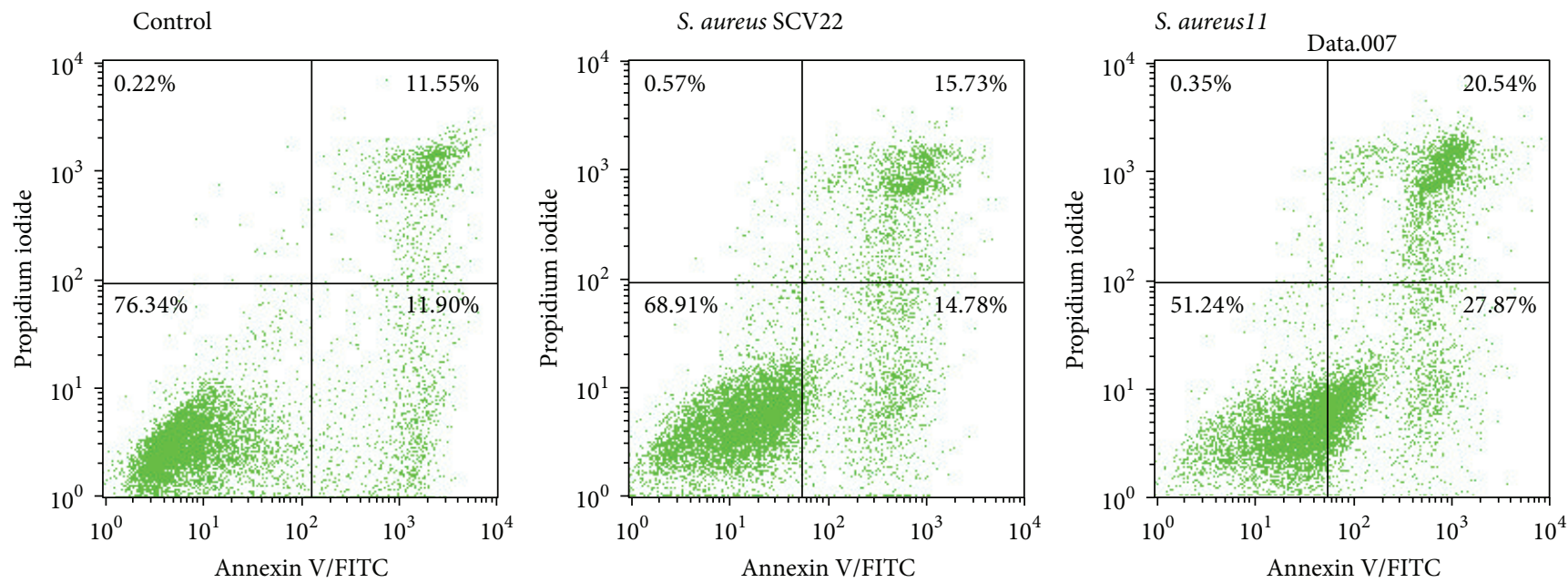

(a)

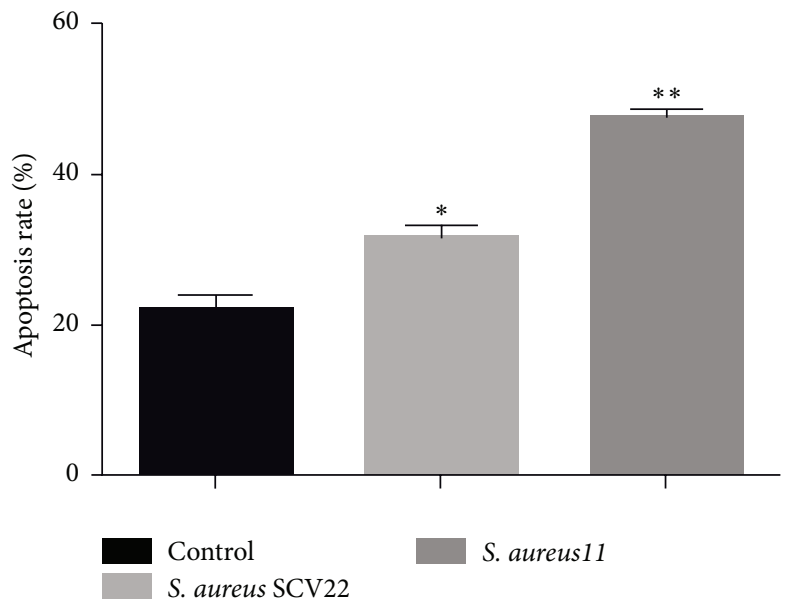

(b)

FIGURE 7: Small colony variants S. aureus SCV22 and their parental strain S. aureusll induced apoptosis of primary bovine mammary epithelial cells $(\mathrm{a}, \mathrm{b}) .{ }^{*}$ There were significant differences between $S$. aureus SCV22 and control. ${ }^{* *}$ There is a very significant difference between S. aureus11 and control. 


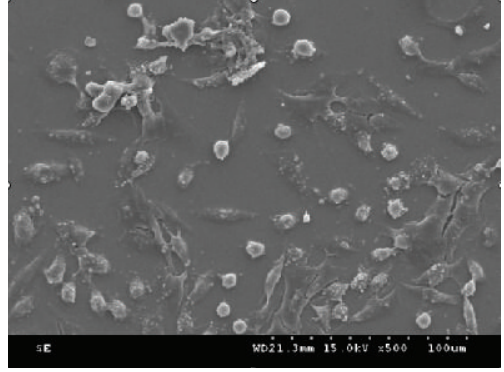

(a)

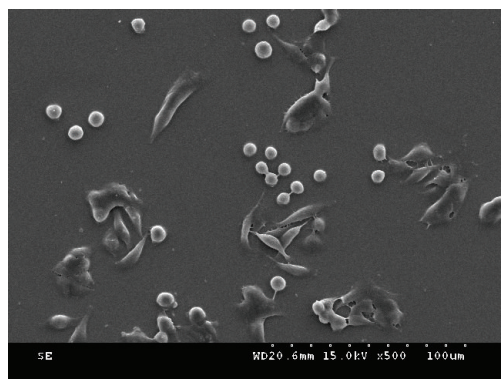

(d)

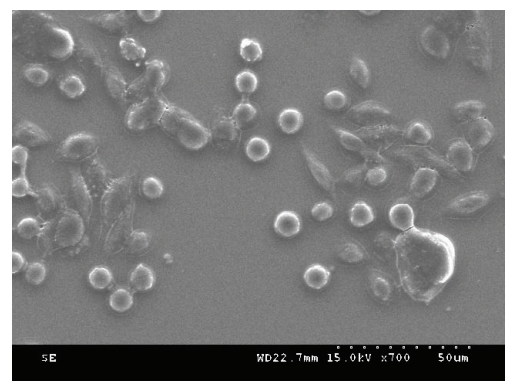

(g)

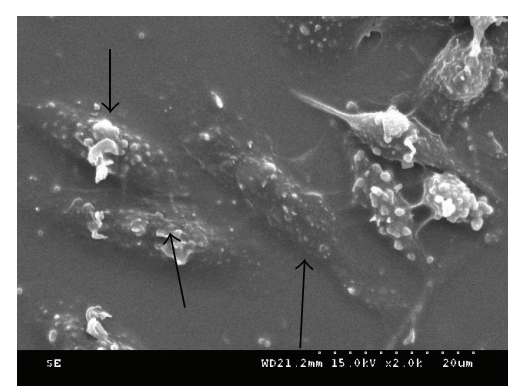

(b)

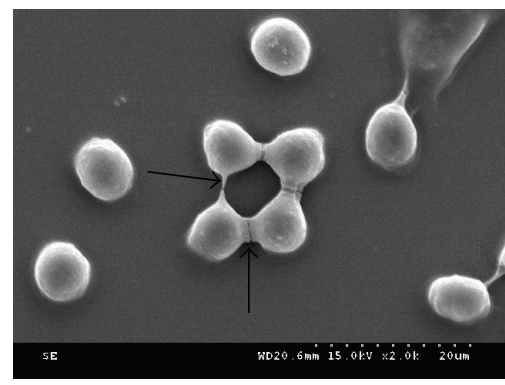

(e)

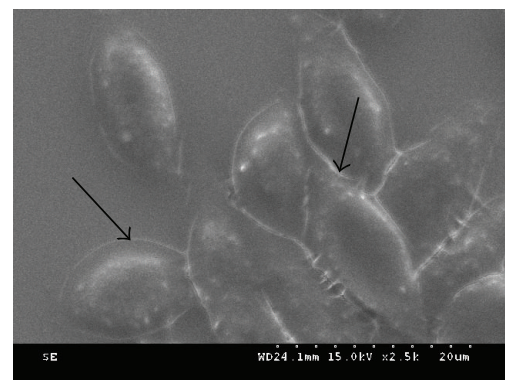

(h)

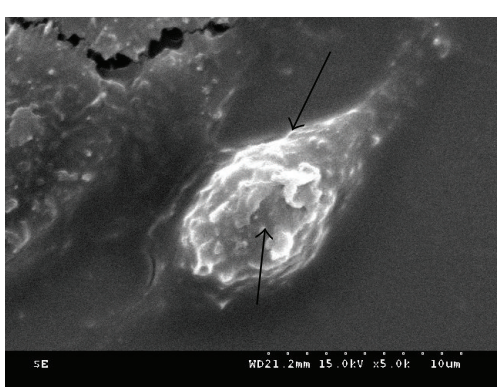

(c)

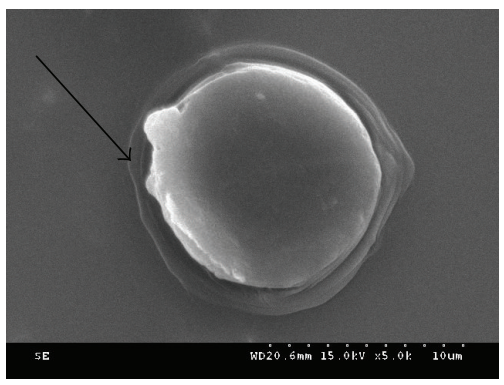

(f)

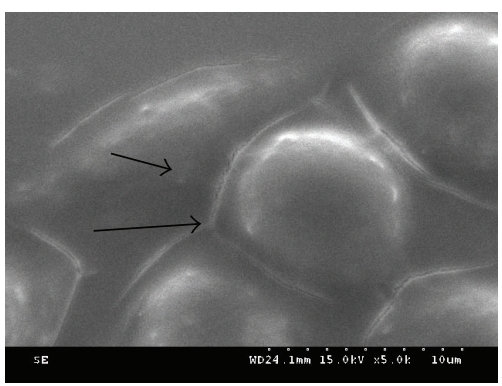

(i)

FIGURE 8: Representative scanning electron micrographs displayed the various effects of apoptosis (controls (g, h, and i), small colony variants S. aureus SCV22 (d, e, and f), and their parental strain S. aureus11 (a, b, and c), 5000x (c, f, and i), 2000x (b, e, and h), and 500x (a, d, and g)). Arrows in (b, c, e, f, h, and i) represented the morphology of cytomembrane.

TABLE 2: Susceptibility test of ATCC 25923 and S. aureus SCV22 isolates.

\begin{tabular}{lcc}
\hline Antibiotic & ATCC 25923 & S. aureus SCV22 \\
\hline Fosfomycin & $\mathrm{S}$ & $\mathrm{S}$ \\
SXT & $\mathrm{S}$ & $\mathrm{R}$ \\
Oxacillin & $\mathrm{S}$ & $\mathrm{S}$ \\
Ampicillin & $\mathrm{S}$ & $\mathrm{R}$ \\
Vancomycin & $\mathrm{S}$ & $\mathrm{R}$ \\
Streptomycin & $\mathrm{S}$ & $\mathrm{R}$ \\
Vancomycin & $\mathrm{S}$ & $\mathrm{S}$ \\
Ampicillin & $\mathrm{S}$ & $\mathrm{R}$ \\
Oxacillin & $\mathrm{S}$ & $\mathrm{S}$ \\
Gentamicin & $\mathrm{S}$ & $\mathrm{R}$ \\
\hline
\end{tabular}

S: sensitive; R: resistant.

SXT: sulfamethoxazole trimethoprim.

production technology, lack of technical personnel, and abuse of antibiotics. In the study, S. aureus SCVs isolated from dairy cows were reported in Yunnan for the first time, which are strongly connected with chronic mastitis [23-25]. To date, there is no treatment to exhaustively eliminate chronic mastitis in production; therefore, further study should be carried out to better know the infection mechanisms of $S$. aureus SCVs in chronic mastitis.

\section{Conflict of Interests}

There is no conflict of interests.

\section{Acknowledgment}

This research was supported by the Chinese National Natural Science Foundation Project (31260629).

\section{References}

[1] R. A. Proctor, P. Van Langevelde, M. Kristjansson, J. N. Maslow, and R. D. Arbeit, "Persistent and relapsing infections associated 
with small-colony variants of Staphylococcus aureus," Clinical Infectious Diseases, vol. 20, no. 1, pp. 95-102, 1995.

[2] B. Kahl, M. Herrmann, A. S. Everding et al., "Persistent infection with small colony variant strains of Staphylococcus aureus in patients with cystic fibrosis," Journal of Infectious Diseases, vol. 177, no. 4, pp. 1023-1029, 1998.

[3] O. Vesga, M. C. Groeschel, M. F. Otten, D. W. Brar, J. M. Vann, and R. A. Proctor, "Staphylococcus aureus small colony variants are induced by the endothelial cell intracellular milieu," Journal of Infectious Diseases, vol. 173, no. 3, pp. 739-742, 1996.

[4] M. L. Kaplan and W. E. Dye, "Growth requirements of some small-colony-forming variants of Staphylococcus aureus," Journal of Clinical Microbiology, vol. 4, no. 4, pp. 343-348, 1976.

[5] J. Lannergård, C. von Eiff, G. Sander et al., "Identification of the genetic basis for clinical menadione-auxotrophic small-colony variant isolates of Staphylococcus aureus," Antimicrobial Agents and Chemotherapy, vol. 52, no. 11, pp. 4017-4022, 2008.

[6] C. Kohler, C. von Eiff, G. Peters, R. A. Proctor, M. Hecker, and S. Engelmann, "Physiological characterization of a heme-deficient mutant of Staphylococcus aureus by a proteomic approach," Journal of Bacteriology, vol. 185, no. 23, pp. 6928-6937, 2003.

[7] J. G. Malone, T. Jaeger, C. Spangler et al., "YfiBNR mediates cyclic di-GMP dependent small colony variant formation and persistence in Pseudomonas aeruginosa," PLoS Pathogens, vol. 6, no. 3, Article ID e1000804, 2010.

[8] A. Oliver, R. Cantón, P. Campo, F. Baquero, and J. Blázquez, "High frequency of hypermutable Pseudomonas aeruginosa in cystic fibrosis lung infection," Science, vol. 288, no. 5469, pp. 1251-1253, 2000.

[9] P. G. Quie, "Microcolonies (G variants) of Staphylococcus aureus," Yale Journal of Biology and Medicine, vol. 41, no. 5, pp. 394-403, 1969.

[10] R. A. Proctor, P. van Langevelde, M. Kristjansson, J. N. Maslow, and R. D. Arbeit, "Persistent and relapsing infections associated with small-colony variants of Staphylococcus aureus," Clinical Infectious Diseases, vol. 20, no. 1, pp. 95-102, 1995.

[11] F. Schaaff, G. Bierbaum, N. Baumert, P. Bartmann, and H.-G. Sahl, "Mutations are involved in emergence of aminoglycosideinduced small colony variants of Staphylococcus aureus," International Journal of Medical Microbiology, vol. 293, no. 6, pp. 427-435, 2003.

[12] H. Atalla, C. Gyles, C. L. Jacob, H. Moisan, F. Malouin, and B. Mallard, "Characterization of a Staphylococcus aureus small colony variant (SCV) associated with persistent bovine mastitis," Foodborne Pathogens and Disease, vol. 5, no. 6, pp. 785-799, 2008.

[13] M. Schneider, K. Mühlemann, S. Droz, S. Couzinet, C. Casaulta, and S. Zimmerli, "Clinical characteristics associated with isolation of small-colony variants of Staphylococcus aureus and Pseudomonas aeruginosa from respiratory secretions of patients with cystic fibrosis," Journal of Clinical Microbiology, vol. 46, no. 5, pp. 1832-1834, 2008.

[14] P. C. Y. Woo, A. S. P. Leung, K. W. Leung, and K. Y. Yuen, "Identification of slide coagulase positive, tube coagulase negative Staphylococcus aureus by $16 \mathrm{~S}$ ribosomal RNA gene sequencing," Molecular Pathology, vol. 54, no. 4, pp. 244-247, 2001.

[15] H. E. Morton and J. Shoemaker, "The identification of Neisseria gonorrhoeae by means of bacterial variation and the detection of small colony forms in clinical material," Journal of Bacteriology, vol. 50, pp. 585-587, 1945.

[16] R. C. Massey, A. Buckling, and S. J. Peacock, "Phenotypic switching of antibiotic resistance circumvents permanent costs in Staphylococcus aureus," Current Biology, vol. 11, no. 22, pp. 1810-1814, 2001.

[17] B. E. Menzies and D. S. Kernodle, "Site-directed mutagenesis of the alpha-toxin gene of Staphylococcus aureus: role of histidines in toxin activity in vitro and in a murine model," Infection and Immunity, vol. 62, no. 5, pp. 1843-1847, 1994.

[18] R. A. Proctor, C. von Eiff, B. C. Kahl et al., "Small colony variants: a pathogenic form of bacteria that facilitates persistent and recurrent infections," Nature Reviews Microbiology, vol. 4, no. 4, pp. 295-305, 2006.

[19] A. Sabat, N. Malachowa, J. Miedzobrodzki, and W. Hryniewicz, "Comparison of PCR-based methods for typing Staphylococcus aureus isolates," Journal of Clinical Microbiology, vol. 44, no. 10, pp. 3804-3807, 2006.

[20] F. Kipp, B. C. Kahl, K. Becker et al., "Evaluation of two chromogenic agar media for recovery and identification of Staphylococcus aureus small-colony variants," Journal of Clinical Microbiology, vol. 43, no. 4, pp. 1956-1959, 2005.

[21] R. W. Lacey and A. A. Mitchell, "Gentamicin-resistant Staphylococcus aureus," The Lancet, vol. 294, no. 7635, pp. 1425-1426, 1969.

[22] J. B. Lyczak, C. L. Cannon, and G. B. Pier, "Lung infections associated with cystic fibrosis," Clinical Microbiology Reviews, vol. 15, no. 2, pp. 194-222, 2002.

[23] F. Kipp, K. Becker, G. Peters, and C. Von Eiff, "Evaluation of different methods to detect methicillin resistance in smallcolony variants of Staphylococcus aureus," Journal of Clinical Microbiology, vol. 42, no. 3, pp. 1277-1279, 2004.

[24] R. W. Lacey, "Dwarf-colony variants of Staphylococcus aureus resistant to aminoglucoside antibiotics and to a fatty acid," Journal of Medical Microbiology, vol. 2, no. 3, pp. 187-197, 1969.

[25] G. Lina, Y. Piémont, F. Godail-Gamot et al., "Involvement of Panton-Valentine leukocidin-producing Staphylococcus aureus in primary skin infections and pneumonia," Clinical Infectious Diseases, vol. 29, no. 5, pp. 1128-1132, 1999. 

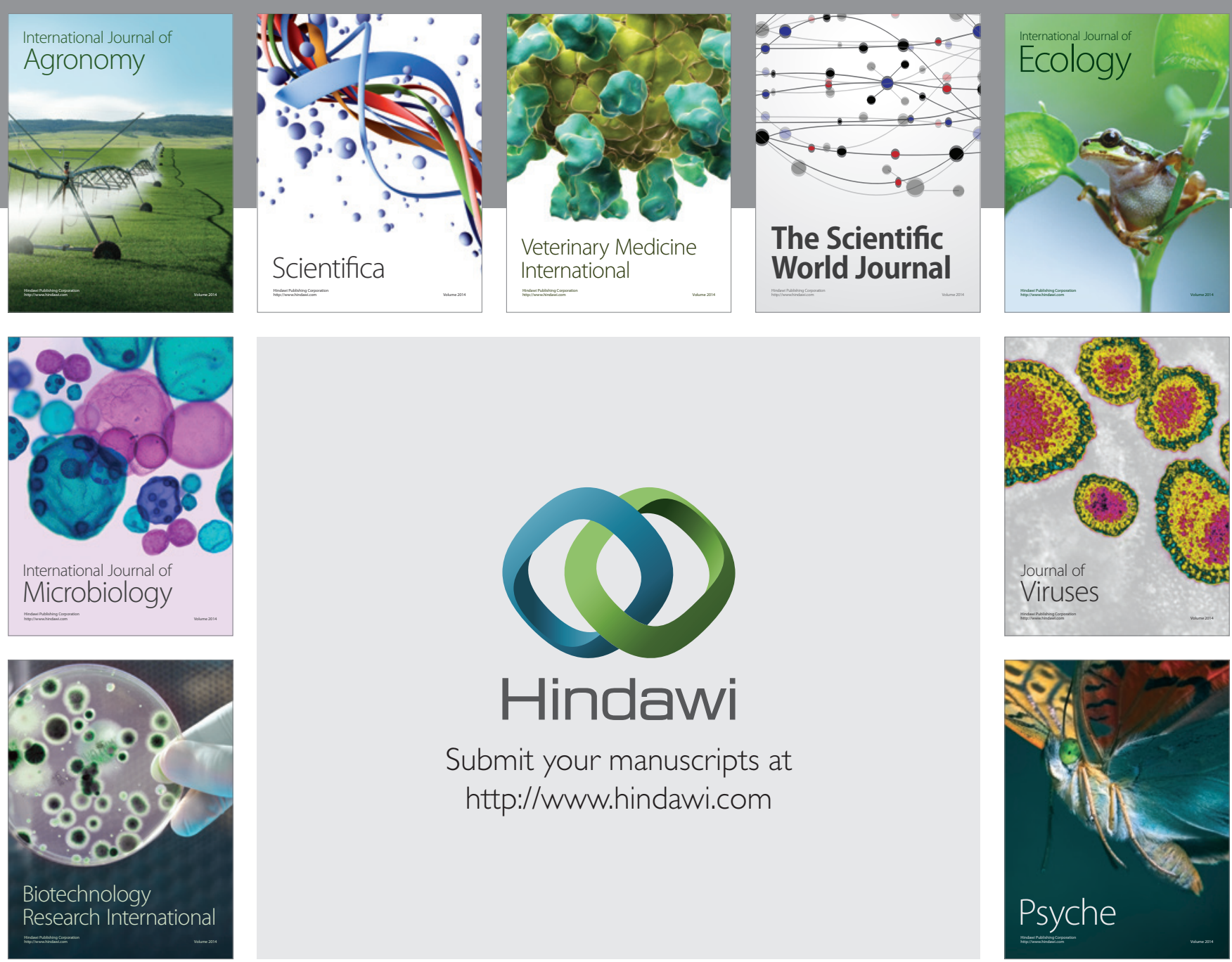

Submit your manuscripts at

http://www.hindawi.com
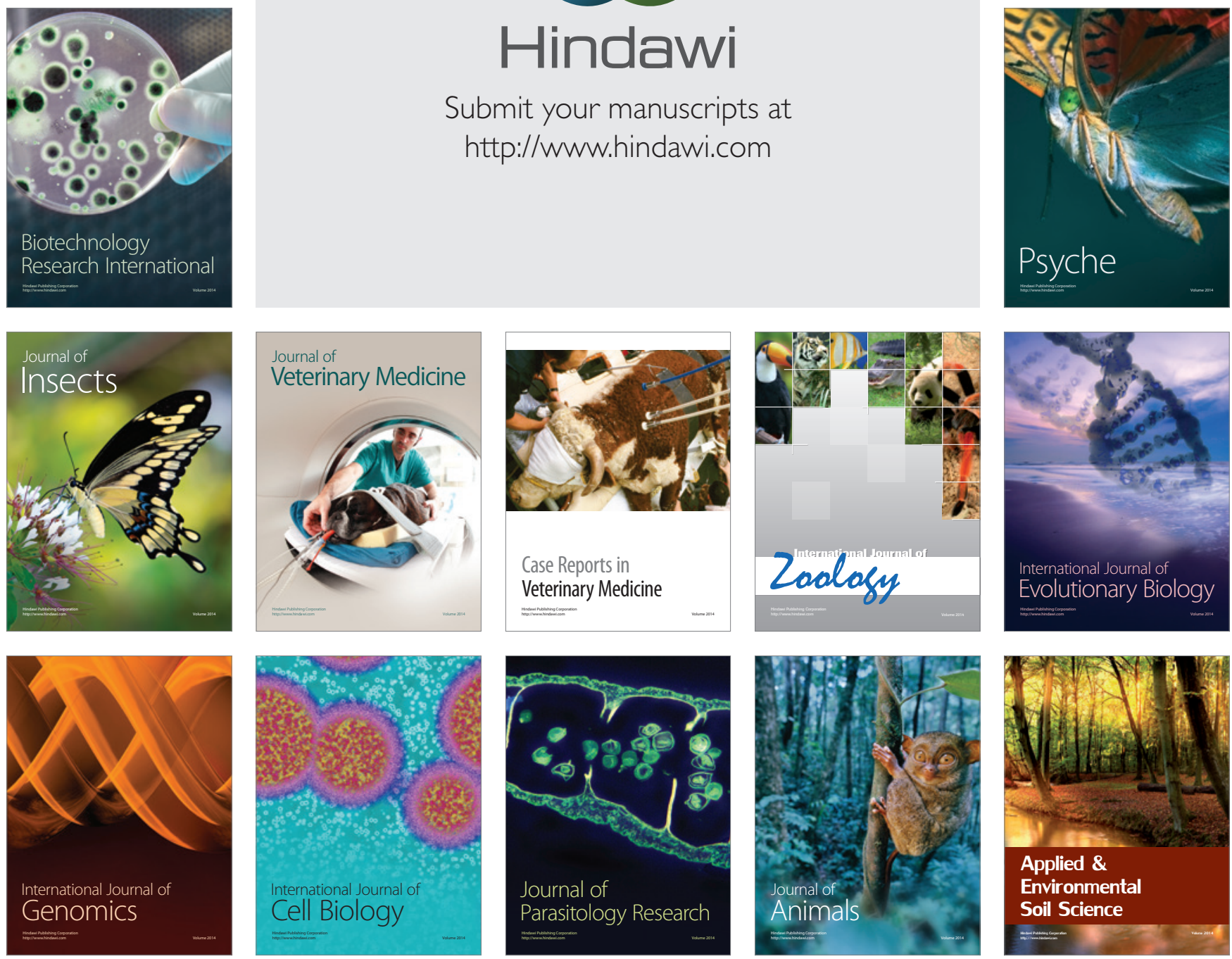